\title{
Research on Tea Processing Technology and Mechanical Motion Mechanism for Fried Tea Machine
}

\author{
Tan Heping $^{1, a^{*}}$, Xu Haiwei $^{1}$, Tang Jiangwen ${ }^{1}$ and Cao jiangping ${ }^{1}$ \\ ${ }^{1}$ Nation Institute of Measurement and Testing Technology, Chenghua District, Yushuang road no.10, \\ Chengdu city, Sichuan province, China, 610056 \\ a49653172@qq.com
}

Keywords: Machining process analysis, Mechanism of mechanism motion analysis, Fried tea machine, Tea machinery processing test.

\begin{abstract}
Traditional way of tea processing have staff labor intensity, low efficiency, high cost, product quality is not standardized, and mechanical processing instead of manual tea with tea very good to solve the above shortcomings, this article from mechanical processing technology of tea, Analyze tea frying machine of working principle and motion mechanism, it is concluded that the working principle of tea frying machine, determines the basic action of eccentric Fried tea institutions, regulating mechanism of elevation and pressure regulating mechanism, and has made the analysis to these institutions. Determine the basic requirement when it begin to processing tea. Finally, through the operation of a prototype test shows that, the fried tea machine motion trajectory and processing Mao-jian tea required basic action is consistent with the conclusion.
\end{abstract}

\section{The Preface}

Traditional tea processing are based on manual operation, in the long-term practice, for a set of tea production experience, but the manual processing there is a tea the intensity of labor is big, low production efficiency, high cost, tea processing, staff technical differences, difficult to make the product quality through the standardization. Need to study on the traditional processing technology, make tea machinery processing technology, and to determine the mechanical processing tea requirements of the basic movements.

\section{The Tea Processing Technology}

Traditional Tea Processing Technology. As we all know, the standard of fresh tea leaves as one bud two Ye Chu exhibition, at the beginning of processing technology for filming, cool breeze, knead, fry green, after rubbing, Fried three green, bedding, milli pan eight procedure [1].

Fresh leaves after back, stand to put 6-12 hours, and then to overcome, pushes the tea again after a cool breeze, to come loose hot, prevent boring yellow, after early appropriate massage, "fry green" into the pot, then through after rubbing, and "three green" into the pan, fry the tea eggs to hands, to do a line and do article also in the pot, the method is both hands tea on palm, using palm force back rub, rub while scattered, make tea billet scattered along the pot wall roll, so repeated many times, works for tea at eighty percent, into the mention hao pan, saute until Bai Hao, tea, sending out of the pot to collect.

Tea Machinery Processing Technology. Through the analysis of the traditional processing technology of tea, you can specify the tea machinery processing technology [2-5].

(1) Fresh leaves out put, fresh leaves after screening should be spread out in a timely manner. Thin folded in her seat or clean on the cement floor, stand put $4-5 \mathrm{~cm}$, the thickness of once every $2 \mathrm{~h}$, lay time 6 to $8 \mathrm{~h}$. To qualitative soft leaves, leaf color out fresh green dark green, fresh leaf weight loss rate was $12 \%$.

(2) Feats, filming for the fresh leaves of booth after put, pan temperature control between $115-120{ }^{\circ} \mathrm{C}$, the speed control in the $60 \mathrm{~s} \mathrm{r} / \mathrm{min}$. According to the tea frying machine production standard evenly into fresh leaves, pushes the time in 2 to $3 \mathrm{~min}$, with fresh leaves lose luster, soft, 
hand knead dough and slightly elastic, no grass for moderation. Filming for the fresh leaves to immediately after cooling, cooling thickness $1-2 \mathrm{~cm}$, open the electric fan, lower leaf.

(3) Rolling, according to the tea frying machine is cast into the steamed leaf quantity requirement, rolling pressure to master the principle of "light - weight - light", control the speed of $120 \mathrm{r} / \mathrm{min}$, the pot temperature control between $115-120^{\circ} \mathrm{C}$. At the beginning of high-grade tea kneading degree appropriate light or no pressure, medium and low-grade tea appropriately increase pressure. High-grade tea rolling time 6-10 min, medium and low-grade tea 15-20 min, rolling to tea gathering, beaded, tea juice tip into a rate of $95 \%$ the scripture, can block off the plane solution. Early rubbing is overweight, can knead tight article tea, but tea juice was attached to the leaves, make the tea poly-phenol was oxidized easily, chlorophyll magnesium discoloration, into a tea color gray.

(4) Fry dried, will manage the Fried dry tea, after the pot temperature control about $80{ }^{\circ} \mathrm{C}$, the speed control in the $120 \mathrm{r} / \mathrm{min}$, Fried dry time 8-10 min, and water content of 5-6\%.

Mao-jian tea have many variety, machining process is roughly same, pan mild processing time can be adjusted according to the actual situation. The same kind of Mao-jian tea in different seasons by picking fresh leaf quality is different, also should adjust according to actual situation.

\section{Tea Frying Machine Working Principle and Mechanism Analysis}

Tea frying machine as a machine, is not a simple piece together a few parts, but an organic whole, the movement relationship between components is very important, is the key to tea frying machine can successfully developed.

\section{Tea Frying Machine Working Principle.}

Basic Structure. The Fried tea machine consists of motor, reducer, elevation adjustment mechanism, pressure regulator, eccentric Fried tea brush, fry, fry tea pot etc and parts, as shown in Fig. 1.

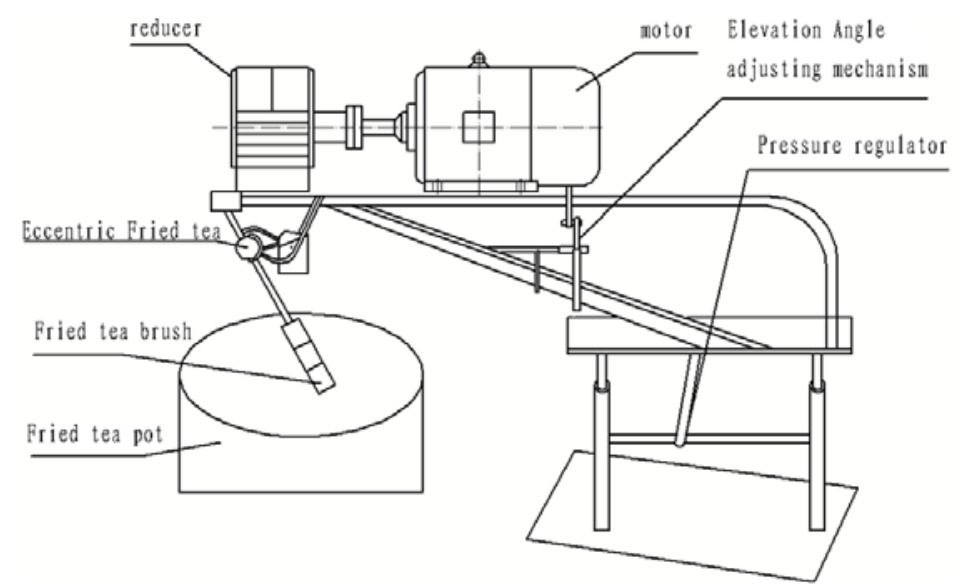

Fig. 1 The structure of tea processing machine.

Working Principle. The machine simulation artificial Fried tea basic movements, powered by motor, Fried tea arm top through the ball joint connection in eccentric sleeve, Fried tea arm of the upper plate, into an organic whole repeatedly through the ball joint and motor rotation when eccentric arm, Fried tea arm to achieve a lower plane circular motion; Fried tea, because of the limitation of positioning baffle rotates, Fried tea brush cannot occur in the process of do circular motion, brush Fried tea and tea interface moment in change, turn every week tea brush with speculation and the circumference of a circle. When Fried tea arm bottom plane circular running, the article in editing, and Fried dry machining process was carried out on the elevation adjustment, Fried tea at the bottom of the arm movement can achieve space circular arc movement, formed up actions; When rolling processing working procedure, no elevation adjustment, pressure regulation, to guide pin to adjust up and down, with guide pin connection plate frame, motor, such as Fried tea arm with guide post moves up and down, and at the bottom of the pan Fried tea arm the distance change, Fried tea brush bending deformation, the elastic function can obtain different pressure acting on the tea, the tea rolling. Can be 
circular motion, the equivalent of a ball joint, then Fried tea in the tea between the brush and the surface of the pot, fired tea rolling brush produced by pressure; Richard and Fried dry processing, without pressure, Fried tea without pressure between the brush and the surface of the pot.

Fried Tea Machine Analysis. According to the requirements of the mechanical processing of tea process, determine the filming for the implementation, rolling, bar and Fried dry processing operations such as organization, analysis of institutions.

Eccentric Fried Tea Mechanism Analysis. As shown in Fig. 2, eccentric Fried tea institutions run time, eccentric arm connected to the gearbox output shaft, eccentric arm length LOA, in eccentric arm shaft directly below point B fixed a ball joint, when the eccentric arm rotation, the bottom is realized planar circular motion, the bottom of the distance away from the ball joint for LBC, bottom can get the biggest radius, its relationship as Eq. (1) show.

$$
r_{\max }=\frac{L_{B C} \times L_{O A}}{\sqrt{L_{O A}{ }^{2}+L_{O B}{ }^{2}}} \mathrm{~mm}
$$

At this point, without adjusting the elevation Angle can be Fried area, its relationship as Eq. (2) show.

$$
s_{\max }=\frac{\pi \times L_{B C}{ }^{2} \times L_{(O A}{ }^{2}}{L_{O A A}{ }^{2}+L_{(O B}^{2}} \mathrm{~mm}^{2}
$$

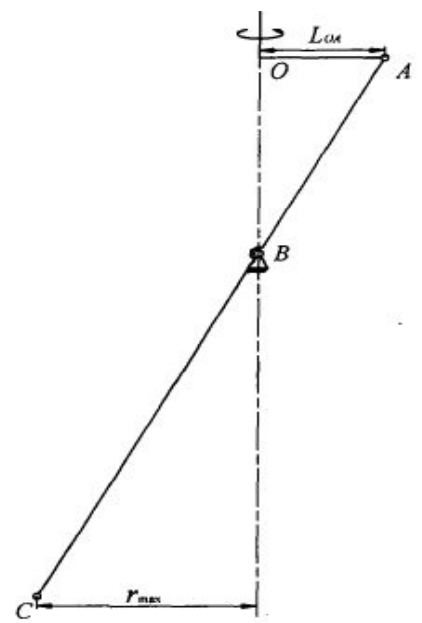

Fig. 2 Eccentric fried tea mechanical motion mechanism.

Elevation Angle Adjusting Mechanism. As shown in Fig. 3, adjust the institutions for elevation of working principle diagram, motor, coupling, reducer, plate, such as Fried tea arm and pin shaft connection between racks, can turn around the pin shaft, realize the elevation adjustment, in order to ensure the motor plate uniform rotation, when the elevation adjustment design of the parallelogram four-bar linkage, when adjusting arm, Fried tea will arm movement before and after movement Angle is 70 angle, at the bottom of the stroke can be reached, its relationship as Eq. (3) show.

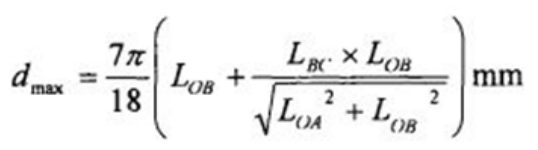




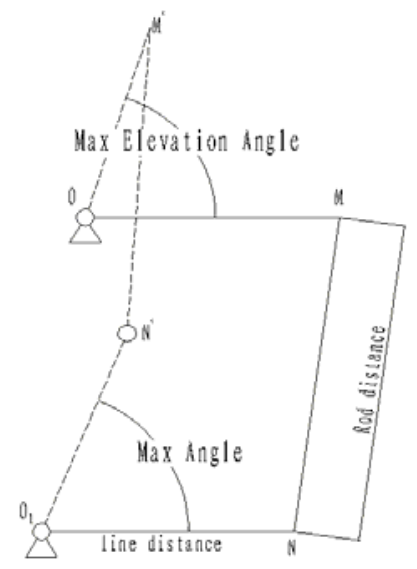

Fig. 3 Elevation angle adjustment mechanical motion mechanism.

Pressure Regulating Mechanism. Pressure is by adjusting the Fried tea brush and distance on the surface of the pan and fry the elastic deformation of brush, mainly to meet different process requirements of different pressure process requirement. Design the guide pin, guide sleeve, machine connected to the guide pin, guide pillar and adjustment, random Fried tea arm moves up and down, brush with Fried tea pot surface distance change the deformation and stress. In filming, and Fried dry processing, guide pin to adjust in a higher position, brush with Fried tea pot surface pressure; In rolling process, the pressure is bigger, guide post regulation in the lower position, so as to get greater pressure. According to the requirements of the traditional process of pressure guide pillar need to adjust the height of the $h$ and the flexibility of Fried tea brush, if elasticity is larger then to adjust the height of the smaller, conversely, the tea every time processing property is different, also different to the requirement of pressure, so I need artificial experience to regulate, $h=40 \mathrm{~mm}$ or so commonly.

\section{Fried Tea Processing Test}

Motion Simulation. Using Pro/Engineer software, according to the functional requirements of the design of various parts, the first part of the installation, and finally the whole A mechanical assembly, the assembly process is set up by the actual movement of the type of motion, set the servo motor Mechanism can exercise, carry out interference check. In case of interference, it is shown that the interference area, and then the size of the parts are modified to check again. Checking until no interference occurs, to provide reference for the manufacture of the prototype.

Test Equipment. Manufactured a prototype for tea processing test. In the test process of the tea, by processing Mao-jian tea experience of experts to carry out operation, pressure, elevation, temperature also by the relevant personnel regulation (Guzhang Mao-jian tea with machining tests).

The main equipment: a common pot, fried tea machine (including tea brush), basket seats, wood etc.

Test Method. (1) Preparation: first to clean the pot, heating to 115 to 120 DEG C, pour a little cooking oil, coated with a cloth to the pot noodles, repeated 3 times, continue to heat $1 \mathrm{a} Z \mathrm{Zmni}$, then pan surface is smooth, can be the next step in the process.

(2) Fixing: to keep the pot temperature, start the machine, motor drives the fried tea rotary brush, speed 60R / min, fried tea brush with the pot surface just contact; the amount of fresh leaves into the pot, fried tea brush driven tea do circular motion, elevation by manual adjustment, after running about 3mni, fixing complete.

(3) Spread cool: to cut off the power supply of the motor, fried tea brush stops rotating, tea after the tea quickly take out, to prevent the burn and amortized in the basket on the table or clean the cement floor, the book, the better, it has been beneficial to the heat dissipation.

(4) Rolled: tea is completely cold down after, for kneading, the tea back into the pot, and speed; 120r/mni, then need to adjust the pressure, according to the experience of tea pressure, in order to prevent the tea for a long time in high temperature zone, the elevation adjusting and pressurized alternately. After about six 10mni, rolling finish. 
(5) Deblocking: quickly take out after rolling tea, then tea into a block can manual deblock, block spread cool until cooling tea leaves.

(6) The rationale, fried dry: the temperature of the pot is maintained at 80 degrees Celsius, the solution of the pot into the pot, the speed of $120 \mathrm{r} / \mathrm{mni}$, elevation by artificial experience. After about 5 a $6 \mathrm{~min}$, the tea can be out of the pot to cool. At this point, the processing of tea processing.

Tea Processing Test Results and Conclusions. According to the Guzhang Mao-jian tea experts (visual and taste), the fried tea machine with Guzhang Mao-jian tea processing requirements. Processing of tea appearance is fine seedlings show, tight knot cord, color green run, liquor color clear, cool taste of alcohol, and meet the requirements of Mao-jian tea shape, color, aroma, taste and liquor color, and as shown in Fig. 4.

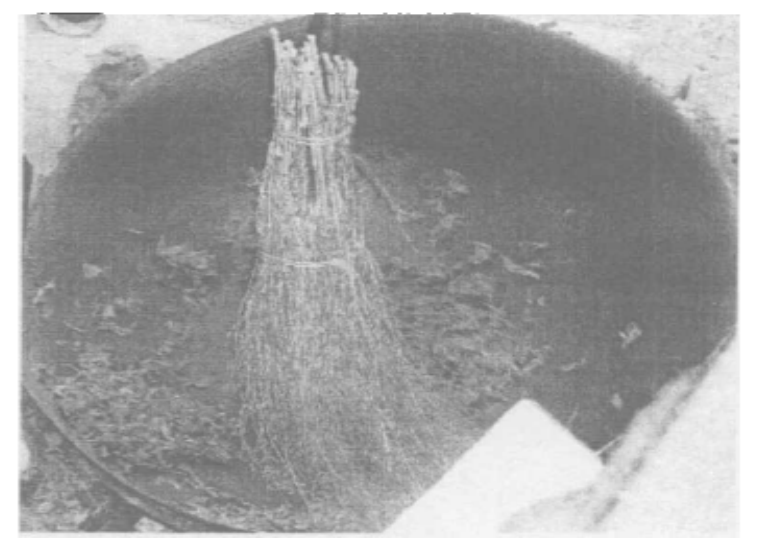

Fig. 4 The picture of shaqing proeess.

\section{Summary}

Based on the analysis of the basic mechanical processing tea movement, it is concluded that the working principle of tea frying machine, determines the basic action of eccentric Fried tea institutions, regulating mechanism of elevation and pressure regulating mechanism, and has made the analysis to these institutions. Determine the basic requirement when processing tea.

Finally, through the operation of a prototype test shows that, the fried tea machine motion trajectory and processing Mao-jian tea basic movements are consistent, the machine of Mao-jian tea processing is feasible, for the design and improved tea frying machine provides a theoretical basis.

\section{References}

[1] J. C. Hu, The types of tea green tea article - ancient zhangs Mao-jian tea, Tea Classics, 26(5) (2005) 137.

[2] L.Z. Lv, Q.P. Zou, Y. L. Qi et. al. Xingyang Mao-jian tea machinery processing technology, Henan agricultural science, 2 (1999) 39-40

[3] H. Jiang, The processing technology of Mao-jian tea, Chinese tea, 2005, pp. 78.

[4] Olajuwon, D. J. Ni, The processing technology and famous quality green tea aroma formation correlation research progress, Silkworm Tea Commun. (2005) 28-29.

[5] C. Zhou, Needle tea mechanism technology research and the discussion on several problems, Silkworm Tea Commun. 4 (2005) 21-22. 